\title{
Simultaneous two-color, two-dimensional angular optical scattering patterns from airborne particulates: scattering results \& exploratory analysis
}

\author{
Stephen Holler ${ }^{\mathrm{a}, *}$, Stephen D. Fuerstenau ${ }^{\mathrm{b}}$, Charles R. Skelsey ${ }^{\mathrm{a}}$ \\ ${ }^{a}$ Department of Physics 83 Engineering Physics, Fordham University, 441 E. Fordham Road, Bronx, NY 10458 \\ ${ }^{b}$ SDF Consulting, San Mateo, CA
}

\begin{abstract}
Light scattering from non-spherical particles and aggregates exhibit complex structure that is revealed only when observed in two angular dimensions $(\theta, \phi)$. However, due to variations in shape, packing, and orientation of such aerosols, the structure of two-dimensional angular optical scattering (TAOS) patterns varies among particles. The spectral dependence of scattering contributes further to the observed complexity, but offers another facet to consider. By leveraging multispectral TAOS data from flowing aerosols, we have identified novel morphological descriptors that may be employed in multivariate statistical algorithms for "unknown" particle classifcation.
\end{abstract}

Keywords: scattering; aerosols; pollen; multivariate analysis; principal component analysis; discriminant function analysis; classification

2010 MSC: 00-01, 99-00

\section{Introduction}

Light scattering is one venerable technique that has been applied to the characterization of aerosol particles. Airborne particulate matter is generally irregularly shaped, 5 and may be comprised of individual particles of dust, minerals, pollen, spores, etc., or aggregates of the same. 1] Due to the diversity of fields in which light scattering from non-spherical particles has been employed, such as urban pollution, 2, 3, atmospheric chemistry, 4, 5] biolog10 ical warfare agents, [6, 7, and biological cell \& flow cytometry analysis, [8], it is important to characterize and understand their properties based on as much information as possible. In developing a cogent understanding of their properties, the mitigation of adverse effects due to their 15 presence becomes possible.

Due to the complex surface morphology of irregularly shaped single particles and aggregates of particles, observation of the rich structure generated by light scattering requires looking beyond the traditional measurement ap20 proach which employs a single (polar) scattering angle. Two-dimensional angular optical scattering (TAOS) offers a means for recording both azimuthal and polar scattering information and thus providing a broader view of the rich interference pattern. 9, 10] The TAOS approach also 25 permits the study of the impact of phase changes and aerosol formation on scattering from liquid media containing inclusions. 11, 12, 13, 14, 15, 16, Due to the spectral dependence of the optical size, the observed pattern changes

\footnotetext{
${ }^{*}$ Corresponding author

Email address: sholler@fordham.edu (Stephen Holler)
}

as the illuminating wavelength is varied; a shorter wavelength results in a denser scattering pattern. 17.

In what follows, we will present our results demonstrating simultaneous acquisition of TAOS patterns at two visible wavelengths from flowing aerosols using a single color CCD camera. These multispectral TAOS patterns were subsequently analyzed to identify for descriptive parameters that might be used as inputs for a multivariate analysis and classification scheme. In addition to the TAOS apparatus (Section 2), we will also describe the morphological descriptors we chose (Section 3), briefly describe the multivariate statistical algorithm (Section 4), and a limited exploratory statistical analysis to assess the potential of these descriptors for use in future work (Section 5 ).

\section{TAOS Apparatus}

We performed TAOS measurements simultaneously using two separate, vertically polarized $\mathrm{cw}$ diode laser sources $(30 \mathrm{~mW}$ at $532 \mathrm{~nm}$ and $70 \mathrm{~mW}$ at $660 \mathrm{~nm})$ and a three CCD chip RGB camera (JAI Pulnix CV-M9 CL, 1024x768 pixels, 24 bit) to record the results. The wavelengths of the diodes were chosen so that there was no overlap with the spectral filters of the CCD camera. As a result, the color channels of the CCD recorded only the scattering at their respective wavelengths. Three separate chips eliminates the Bayer filter used in single chip RGB cameras thereby assuring that each color has maximum spatial resolution. Since we only operated red and green lasers, we used the blue channel as a background measurement. Our setup was careful to eliminate stray blue light, so the noise was principally due to electronic read noise, which is taken to 
be uniform on each CCD chip. The blue frame, in its entirety, was subtracted from both the red and green frames on a 1-to-1 pixel basis to remove this background noise prior to any data processing.
(A)
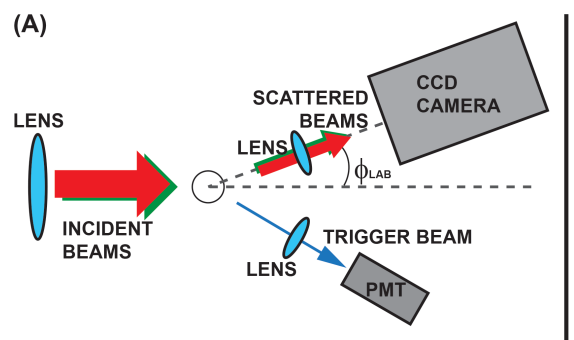

(B)

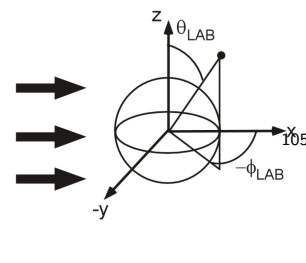

Figure 1: (A) Schematic of the light scattering configuration. The CCD camera and trigger PMT are not located within the aerosol chamber. The collinear lasers enter from the left and focused to the sample region circle with the first lens. Particles are injected into the sample chamber through a nozzle at the top of the chamber; flow is directed into the page. The scattered light is collected by the small lens and recorded by the color CCD camera positioned at the upper right. The PMT located at the bottom collected scattered signal ${ }^{115}$ and triggered the CCD camera to record the TAOS pattern. (B) The laboratory geometry defining the angles reported in the TAOS data.

A schematic of the experimental light scattering configuration is shown in Fig. 1(A). Figure 1(B) shows the ${ }^{120}$ 65 laboratory scattering frame and the geometry for the angles reported.

The core of the aerosol chamber was constructed from a single block of aluminum with several inlet and outlet ports. These ports were sealed with windows to maintain

70 a good airtight seal when the lid was secured in place. ${ }_{125}$ The two lasers were combined externally using a dichroic beamsplitter, making them collinear. The combined beam was injected into the chamber via the inlet port on the left. After traversing the sample region, the residual beam was directed out of the chamber and into a beam dump in order to minimize background scattering. The scattered ${ }^{130}$ light was collected and directed out of the chamber to the CCD camera through a port in the near-forward direction. Additional optical components and ports were included in the chamber design for laser induced fluorescence measurements, and were not used in the present work.

A nozzle is located in the lid and positioned above the sample region. An exhaust port is located at the base of the chamber to draw the aerosol stream out. A diaphragm pump is connected to the exhaust of the chamber, partially evacuating it. Consequently, air is drawn through the noz-140 zle into the chamber. To constrain the aerosol particles, a tower was constructed above the inlet nozzle. For these experiments, the apparatus was operated in a Class 1000 soft-walled clean room so only those aerosols we were interested in studying were observed. A cloud of particles was ${ }^{145}$ introduced approximately $1.5 \mathrm{~m}$ above the nozzle. No special steps were taken to prevent aggregation within the particulate cloud, and aggregate formation was not inferred
Powdered aerosols were "puffed" into an opening at the top of the tower and allowed to settle. Those close to the nozzle were drawn into the sample chamber. Upon exiting the nozzle the particles were directed into the sample volume where they were illuminated by the red and green lasers. The laser beams were gently focused to approximately $100 \mu \mathrm{m}$ spot size. The scattered light was collected by a small lens having a focal length $f=14 \mathrm{~mm}$ situated with its optic axis $20^{\circ}$ away from the forward direction of the combined laser beams and subtending $\pm 11^{\circ}$. This small lens was affixed to a post in order to eliminate any obstruction of the forward scattered beam by a lens mount. The lens is configured in the Abbé sine condition. In this configuration, a small region of the object plane near the optic axis of the system is imaged sharply despite the angular divergence of rays entering the imaging system. [18] The advantage of the Abbé sine condition is that it provides a convenient means of transforming the scattered angle into a linear displacement across the face of the array detector. 9. According to the Abbé sine condition and the geometric configuration of the components, this yields a collection angle of $\pm 11^{\circ}$ (in the laboratory reference frame) 10] This amounts to collection of scattered light, in the lab frame (Fig. 1(B)), in the ranges $9^{\circ}<\phi_{L A B}<31^{\circ}$ and $79^{\circ}<\theta_{L A B}<101^{\circ}$. The CCD camera was triggered using a signal recieved by a photomultiplier tube monitoring through a separate port in the chamber(Fig. 1(A)). After triggering, the TAOS signal was acquired and the data recorded to disk.

\section{Image Processing \& Morphological Descriptors}

Previous attempts to classify aerosol particles based on their TAOS patterns employed descriptors (e.g., spatial frequency components) for the multivariate algorithm that did not reflect particle morphology. [19, 20, Other attempts have been made to classify unknown scatterers based on a priori classes of model particles, 21 and the application of inverse methods to use TAOS data to reconstruct particle morphology. 22] Previous research characterizing unknown aerosols by TAOS employing morphologically relevant descriptors has been limited to asymmetry parameters. 23, 24 In this present work, we sought to extend the morphological descriptors and identify those that we felt reflect the physical characteristics of the particle under study. The TAOS images were processed in MatLab and morphological descriptors were obtained for the classification routine. These physically relevant descriptors leverage the two-color TAOS data obtained from the aerosol scattering and served as the inputs for the classification routine. The four features applied to the two color channels (red \& green), which led to seven morphological descriptors for the classification algorithm, were

- Mean Intensity

- Watershed 


\section{- Entropy}

Mean Intensity. The integrated scattered intensity level over a given solid angle yields an observed signal that correlates with particle size. Given two similar aerosol particles of different sizes, the larger particle would have $\mathrm{a}^{210}$ reater scattering cross-section resulting in more light being scattered in a given solid angle than the smaller particle. This greater scattering signal spread over the same number of accumulated pixels on the CCD camera would result in a greater mean value for the image, thus serv$\mathrm{ng}$ as a rough indicator of particle size. For this feature extraction measure, a two-dimensional average is taken of 215 the TAOS image after background subtraction.

The morphological descriptor generated from the mean intensity value is the ratio of the average green channel signal to the average red channel signal $(<$ green $>/<$ red $>$ ). This was done to mitigate fluctuations in scattered 220 intensity from different particles due to spatial variations in the crossing of the illuminating laser beams.

Watershed. The watershed routine in image processing provide a means for distinguishing or separating features within an image. The algorithm gets its name from the natural tendency of water to collect in basins, which may be separated by local maxima. The WATERSHED function distance to the nearest non-zero pixel value. This provides230 a gradient profile of the image. The gradient, and corresponding local minima provide a means for segmenting the image. These segments are then processed and labeled to determine the number of regions within the image.

The segmentation of TAOS images using the watershed routine follows the same basic principle. However, ${ }_{235}$ some pre-processing steps were implemented before determining the speckle pattern count within the observed solid Due to noise in the image, we performed a smoothing operation (twenty times repeated application of a $3 \times 3$ discretized Gaussian filter) before applying the watershed routine. If such steps were not taken, over-segmentation would occur and no useful information would be obtained. ${ }^{240}$

190 The smoothing process eliminated high-frequency artifacts associated with laser noise, diffraction from optical components, etalon effects, etc.

Once the smoothing operation was complete the image was scaled to range between 0 and 1 . A gray threshold ${ }^{245}$ 195 was computed and the image subsequently converted to a binary image. This image was then processed using the WATERSHED function to compute the number of watershed basins using 8 neighbor connectivity. In some instances, small regions remained that were associated with noise, and did not correspond to scattered intensity from the aerosol particles. These were identified and eliminated to arrive at the number of regions that correspond to scattered signal in the image.
Entropy. In image processing, the term entropy describes 5 a statistical measure of the randomness of an image. This parameter may be used to characterize the texture of the image. Formally, entropy is the expected value of the selfinformation. That is to say that it is a measure of the average amount of information received based on what is produced by the source. It may be interpreted as the measure of the average a priori uncertainty regarding the image.

An image's entropy is quantified according to

$$
S=-\sum_{i} p_{i} \cdot \log _{2}\left(p_{i}\right)
$$

where $p$ is the vector of counts obtained from the histogram of the image divided by the total number of pixels in the image (i.e., $768 \times 1024=786,432$ ). Essentially, it is the probability that the intensity value at a particular bin location will occur in the image.

The speckle pattern inherent to the TAOS images of aggregates, surface roughened particles, and non-spherical particles makes the entropy measure an interesting feature to examine. It is interesting to note that within the region observed, the entropy computed for a spherical particle is low. Its use as a property that characterizes an image's texture makes it wholly applicable to the description of highly textured TAOS images, and evoking a sense of the rough aerosol surface.

Modified Autocorrelation. The modified autocorrelation of the image was chosen to provide a measure of the symmetry of the scatterer. The modified autocorrelation was computed such that the cross-correlation is performed between the image and its spatially flipped counterpart. The correlation coefficient $(r)$ is found using

$$
r=\frac{\sum_{m} \sum_{n}\left(A_{m n}-\bar{A}\right)\left(A_{m n}^{f}-\bar{A}\right)}{\sum_{m} \sum_{n}\left(A_{m n}-\bar{A}\right)^{2}}
$$

where $A$ is the TAOS image, $\bar{A}$ is the mean value of the image, and $A^{f}$ is the flipped image.

Since our apparatus is configured to measure light scattering centered in the polar direction (lab frame, Fig. 11(B)) at $\theta_{L A B}=90^{\circ}$ (i.e., the equatorial plane), light scattering from symmetric particle would exhibit a corresponding symmetry in the data. During the processing a copy of each TAOS image was produced, flipped and then the cross-correlation between the two images was computed. The reasoning being that the scattering pattern from a symmetric particle would look the same when flipped and therefore would be highly correlated, however aggregates and textured particles would break the symmetry leading to a decrease in the measured correlation.

\section{Analysis}

For our analysis we employed routines that are part of MatLab's Statistics Toolbox. This allowed us flexibility in 
exploring the efficacy of the classification using the morphological descriptors discussed in Section 3 . However, 305 for completeness we will provide a brief description of the underlying approach.

The four features discussed above were applied to both color channels and became the inputs for the multivariate algorithm and classifier routine. They produced only seven morphological descriptors since the mean intensity was used ratiometrically so we eliminate any confounding effects due to particle location in the beam and fluctuations in the laser intensity.

The descriptor data was subject to a two-stage analysis: Principal Component Analysis (PCA) followed by Discriminant Function Analysis (DFA).25 The details of the rigorous application of one or both of these multivariate statistical approaches to the analysis and classification of TAOS data, using different descriptors, have been described elsewhere. [19, 20, 26, 27] In essence, PCA serves to reduce the dimensionality of the data set by formulating an orthogonal basis set where the first few principal components contain the majority of the information in the data set.25. The principal components were computed using the PRINCOMP function in MatLab.

Discriminant Function Analysis (DFA), 28, 29, 30 on the other hand, seeks to maximize the separation among the classes. This is done by defining a discriminant function $D_{l}(i)$ that maps the principal component scores

$$
D_{l}(i)=\alpha_{l, 1} t_{l, 1}+\alpha_{l, 2} t_{l, 2}+\cdots+\alpha_{l, A} t_{l, A}
$$

Here $i$ is the index of the TAOS pattern, the $\alpha$ 's are unknown coefficients, the $t_{i}$ 's are the PC scores (taken to be independent variables), and $l$ is the function index, which ranges from 1 to the lesser of $g-1$ (the number of dependent groups -1 ) or $k$ (the number of independent variables). The number of discriminant functions required is less than the number of classes to be discriminated, and, in general, one fewer function is needed. 25, 31

The unknown discriminant coefficients $(\alpha)$ are determined by solving the general eigenvalue problem

$$
(X \cdot W) V=\lambda W V
$$

where $X$ is the Total Sums of Squares and Cross-products matrix, $W$ is the Within-groups Sums of Squares and Cross-products matrix, $V$ is the unscaled matrix of discriminant function coefficients and $\lambda$ is the diagonal matrix of eigenvalues. The solution to Eq. 4 maximizes the distance between the classes.

\section{Discussion}

\subsection{Results}

Aerosols were introduced to the TAOS apparatus via an enclosed chimney, which sat above the nozzle assembly and extended approximately $1.5 \mathrm{~m}$ above the chamber. $\mathrm{A}^{320}$ cloud burst formed near the top of the chimney allowed the particles to disperse and slowly fall towards the instrument. The pump drew aerosol particles into the nozzle assembly and into the sample chamber so that they could be interrogated by the collinear laser beams.
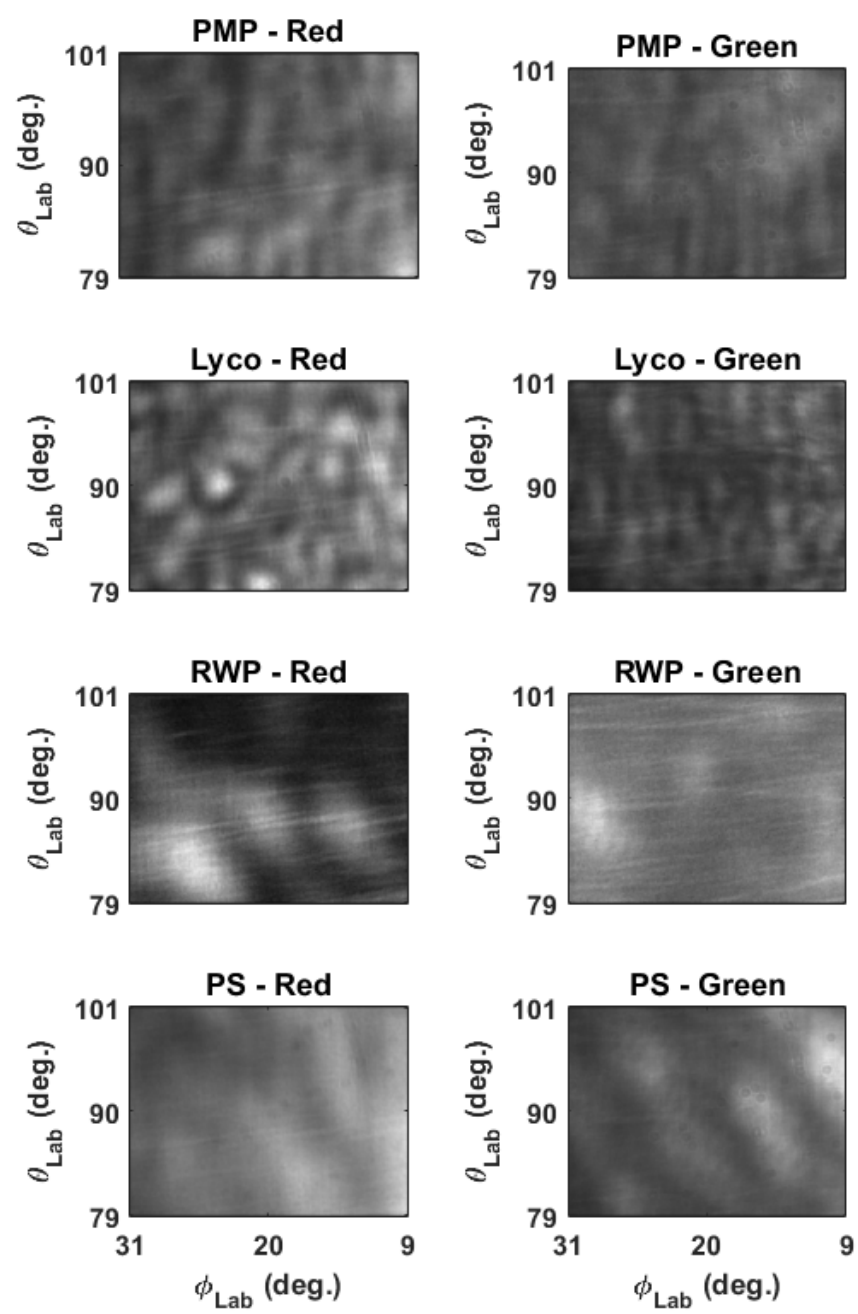

Figure 2: Examples of TAOS patterns recorded in the near-forward direction acquired simultaneously with red and green incident light from different aerosols used in the experiments. These images were processed using the filter algorithm described in the text.

TAOS data was collected from different aerosol particles. The principal classes of particles we looked at were Lycopodium spores (Lyco), Paper Mulberry Pollen (PMP) particles, and Ragweed Pollen (RWP) particles. These pollen particles were selected to demonstrate the ability to classify particles based on their two-dimensional light scattering patterns. Based on the results obtained from of these particles, a database was developed as a means to compare "unknown" TAOS patterns. The database comprised 111 TAOS images representing 26 Lycopodium spore particles, 74 Paper Mulberry pollen particles, 11 Ragweed pollen particles, and 14 Powered Sugar (PS). The PS particles were included as a confounding element for the classification routine. Each class was measured and the data saved for subsequent analysis. Ex- 
amples of TAOS patterns recorded from different aerosols used in the experiment are shown in Fig. 2, These images were filtered according to the algorithm described above.

The rich texture associated with light scattering from rough particles is noted in these images. The Lycopodium spores are approximately round with diameters $\sim 30 \mu \mathrm{m}$ but with concave corrugations in the surface that serve to break up the ring pattern otherwise seen from spherical scatterers. The Ragweed pollen is also approximately round with diameters slightly smaller $(\sim 20 \mu \mathrm{m})$, however, unlike the Lycopodium's, the surface roughness is convex, appearing as small spikes across the particle's surface.

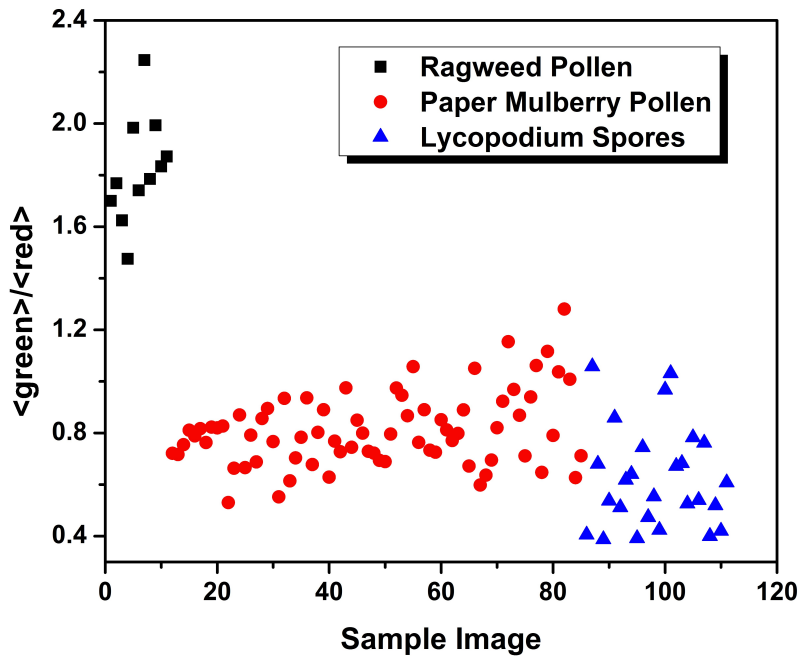

Figure 3: Plot of the intensity values computed from the TAOS images that comprise the database matrix used for the classification scheme.

Figure 3 shows the mean intensity morphological descriptor obtained from those TAOS images that formed the database matrix. This simple metric already shows some separation among the classes observed, with particular distinction being made for the Ragweed pollen (squares) versus the Paper Mulberry pollen (circles) and the Lycopodium spores (triangles).

The watershed routine was applied to all the images in the TAOS database. This routine was used to determine the number of peaks in the images, so before segmenting the image, the TAOS image was inverted to turn peaks into valleys. Since this metric is a measure of the number of speckle islands formed in the observed solid angle and not related to the laser intensity, we used both the red and green channels as morphological descriptors. The results are summarized in Table 1. In all cases the green channel shows greater segmentation of the TAOS pattern (i.e., more islands of intensity in the given solid angle), which is consistent with a larger optical size. The density of peaks also correlates with the aerosol particle's geometric size.

Applying the entropy calculation (Eq. 1) to the same database images yielded the results shown in Fig. 4. The different particle morphologies produced varied results based
Table 1: Summary of the watershed segmentation of the TAOS images used in the classification database showing the mean number of peaks counted in the observed solid angle for the different aerosol

\begin{tabular}{l|l|l|}
\hline classes. & Red & Green \\
\hline Aerosol Type & $15.73 \pm 0.86$ & $16.04 \pm 0.50$ \\
\hline Ragweed Pollen & $12.18 \pm 0.41$ & $15.00 \pm 0.96$ \\
\hline Paper Mulberry Pollen & $10.82 \pm 1.23$ & $12.20 \pm 0.40$ \\
\hline
\end{tabular}

on the texture of their images. Because we are looking at normalized probabilities for particular intensity values in the image we chose to plot the color channels separately. In both channels the separation of the measured entropy among the different particles classes is quite distinct. Note that entropy is expected to have a spectral dependence, particularly where the particle is absorbing. The Lycopodium spores show the greatest variance, but still remain distinct from the Ragweed pollen and the Paper Mulberry pollen.

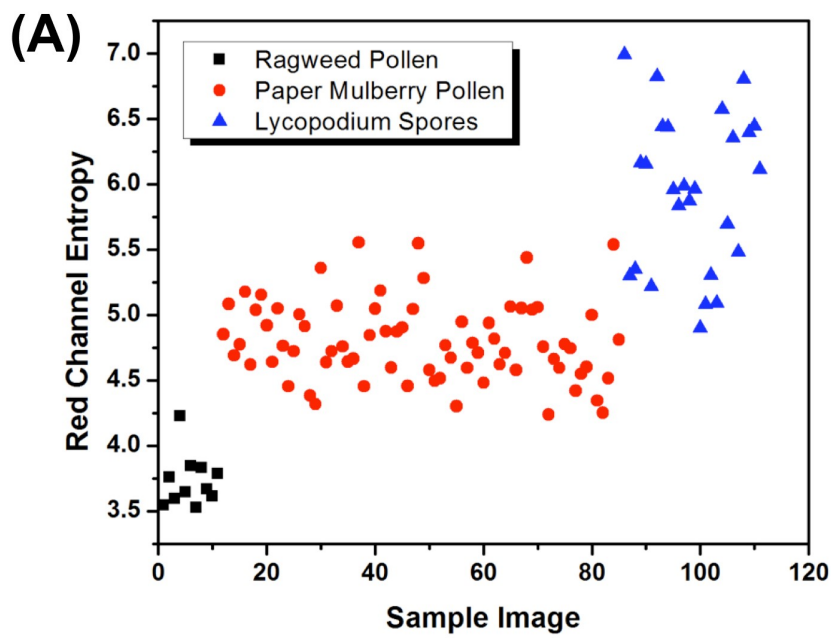

(B)

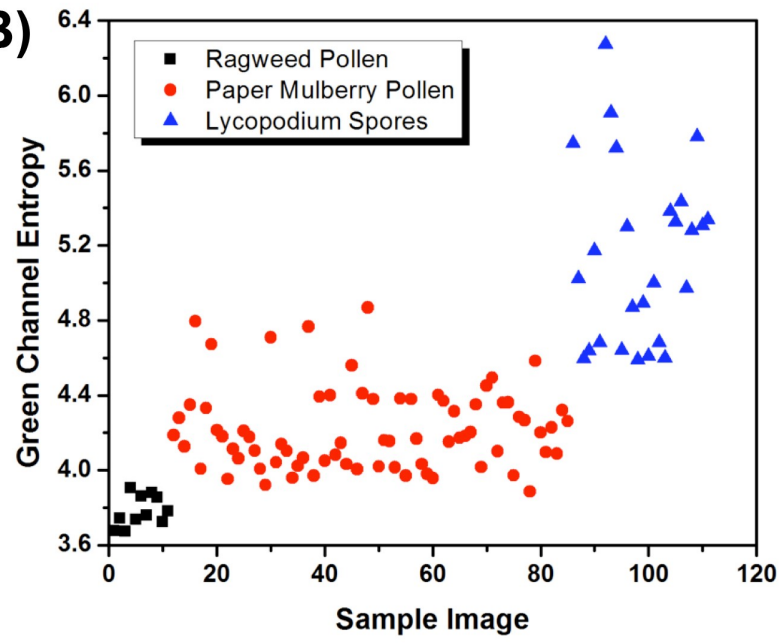

Figure 4: Plot of the entropy values computed from the (A) red and (B) green channels of the TAOS images that comprise the database matrix used for the classification scheme.

The modified autocorrelation computation (Eq. 2) was 
applied to the TAOS images to be used in the classificametric is meant to capture the symmetry of the scattering about the equatorial plane. A highly symmetric particle will have a TAOS pattern that is symmetric about this plane and therefore lead to a high correlation coeffitors, this metric showed weak correlation in the red channel and stronger correlation in the green channel, however the strongest correlation was only around 50\%. The Lyocpodium spores exhibited the greatest similarity among the two channels, but neither was highly correlated. This 425 indicates that, by and large, these particular aerosols were asymmetric scatterers.

Table 2: Summary of the modified autocorrelation results from ananlysis of the TAOS images used in the classification database

\begin{tabular}{|l|l|l|}
\hline Aerosol Type & Red & Green \\
\hline Lycopodium Spores & $0.31 \pm 0.15$ & $0.39 \pm 0.14$ \\
\hline Ragweed Pollen & $0.03 \pm 0.05$ & $0.43 \pm 0.07$ \\
\hline Paper Mulberry Pollen & $0.15 \pm 0.14$ & $0.45 \pm 0.12$ \\
\hline
\end{tabular}

\subsection{Classification}

The discriminant functions (Eq. 3) were used to generate a distribution in the multi-dimensional space formed by the three retained vectors. The decision to retain three principal components was determined by invoking standard practices of considering the relative value of the eigenvalues as well as a scree test on the principal component variances. 25] Both approaches yielded the same number ${ }_{445}$ of components. Based on the cluster of discriminant function scores a centroid for each group was computed. Using the MatLab CLASSIFY function we explored how the classification proceeded using different metrics for computing distance from the group centroid. We found that the ${ }_{450}$ Mahalanobis distance produced the greatest classification accuracy. The Mahalanobis distance is defined as

$$
D_{M}=\sqrt{(x-\mu)^{T} C^{-1}(x-\mu)}
$$

where $x$ is the multivariate vector with a covariance matrix ${ }^{455}$

${ }_{400} C$ and $\mu$ is the mean vector associated with the group. Assignment to a particular group of aerosols was based on the associated minimum of $D_{M}$. Iif the covariance matrix were replaced by an identity matrix, then the Mahalanobis metric would revert to a simple Euclidean distance of the ${ }^{460}$ discriminant score to the aerosol group centroid.

Prior to performing the classification analysis on "unknown" sample data, the reference data that was used to form the database matrix was run through the TAOS Analysis algorithm to determine the extent to which the program successfully classified this known data. In addition to the Lycopodium spores, Paper Mulberry Pollen, and Ragweed Pollen, the database was modified to include features extracted from TAOS images of Powdered
Sugar particles. Due to the high variability of scattered signals that occur in the TAOS images, each class is not represented by a narrow bin, but rather forms a multidimensional region in which some characteristics overlap. This is apparent by examining the plots shown in Fig. 3 and Fig. 4. As a result, one would not expect perfect classification with this routine, but a high degree of accuracy is anticipated. Based on the assignments determined from the minimum Mahalanobis distance (Eq. 5) we found that $84 \%$ of the TAOS patterns were correctly assigned. This provided sufficient accuracy test the classification of "unknown" aerosols against this database.

The "unknown" test samples were taken from the larger set of TAOS data recorded for PMP and RWP particles. In total twelve images were used to run through the classification routine. The morphological descriptors for these twelve TAOS patterns were computed and run through the PCA routine to generate PC scores, which served as inputs for the previously determined discriminant functions. Based on the resulting scores, $D_{M}$ was computed for each and aerosol group assignment was determined. We found that classification was correct $67 \%$ of the time. For PMP four out of seven were correctly assigned, while for RWP the correct assignment was four out of five. When the Mahalanobis metric was used, none of the "unknown" TAOS patterns were assigned to the confounding group associated with powdered sugar. While not perfect, this assignment rate is good; comparable to the results from previous efforts.

The observed classification accuracy was consistent with visual observations of a three dimensional plot of the retained principal components. Figure 5 shows a threedimensional plot of the principal components scores from the TAOS patterns used in the database (including the confounding powdered sugar sample) as well as the scores from the "unknown" test samples. The unknown samples are represented by the black squares (PMP) and black diamonds (RWP). Inspection of the figure shows that four of the seven PMP samples in in the immediate vicinity of the circles (PMP database) while four of the five diamonds lie in close proximity to the upright triangles (RWP database). The stars in Fig. 5 represent the PS database while the inverted triangles correspond to the scores from the Lycopodium spore database. The lone misclassified RWP lies clearly near the centroid of the Lycopodium spores while the three misclassified PMP are close to the RWP centroid. These observations match with the results of the discriminant function classification.

It is worth noting that while our present approach does not supplant previous efforts, [19, 20] and will require more extensive data (i.e, more diverse particles and a greater number of TAOS images) to form a more complete classification database and realize a meaningful comparison of the different morphological descriptors used, the goal of this preliminary study to demonstrate good classification results using descriptors that we felt evoked a sense of the physical and morphological characteristics of naturally oc- 


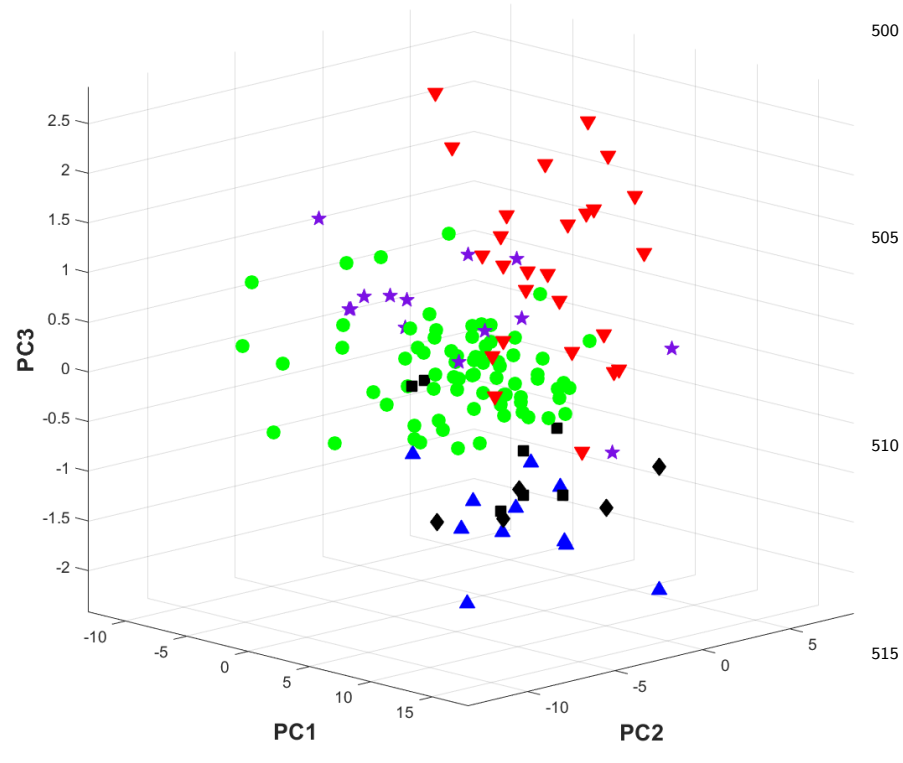

Figure 5: Three dimensional plot of the principal component scores for the TAOS patterns in the database and the "unknown" test sam-520 ples.The database was comprised of PMP (circles), RWP (upright triangles), Lycopodium spores (inverted triangles) and PS sample (stars). The "unknown" samples were obtained from PMP (squares) and RWP (diamonds) aerosols.

curring aerosols has been realized.

\section{Summary}

The classification of unknown aerosol particles based $_{530}$ on their two-dimensional elastic scattering patterns is a daunting task. The rich patterns produced by the nonspherical and rough shapes associated with many common aerosols presents a major barrier in quantitative comparisons among theory and experiment. However, the ap-535 plication of multivariate statistical algorithms to specific

${ }_{480}$ features which characterize TAOS images do provide a means for assigning unknown particles to classes. The results presented have demonstrated the ability to perform such measurements using inexpensive semiconductor ${ }_{540}$ diode lasers and relatively inexpensive CCD arrays. Although the dynamic range of the observed TAOS images is not as great as possible with Q-switched lasers and intensified CCD arrays, adequate results may be achieved for a fraction $(\sim 1 / 10)$ of the price. Furthermore, this TAOS ${ }_{545}$ instrument has incorporated, for the first time, to the best of our knowledge, a means for acquiring multi-color light scattering data simultaneously with a single detector. The morphological descriptors derived from the TAOS images demonstrate utility in classification of unknown aerosols550 within a known database matrix, and can achieve a correct assignment in excess of $80 \%$.

The analysis of the experiments discussed above highlight the possibilities for classification based on multi-spectral TAOS data. The results show that given TAOS patterns555 from "unknown" aerosols reasonable assignment within the database matrix may be achieved. Analysis of the reference data employed in the formation of the database matrix show that the algorithm is good, with greater than $80 \%$, but also indicates that within the database outliers do exist. Improvement of the classification ability can be achieved by eliminating those outliers, providing a tighter region within which the extracted features lie. Correlation of the unknown samples with the database would then provide improved specificity in the class assignment.

The assignment, with $100 \%$ certainty, of naturally occurring aerosol particle's based solely on TAOS image data may not be possible due to a number of effects that contribute to the complex speckle pattern observed from flowing particles. Among the confounding factors are shape and size variability, orientation, aggregation, packing fraction, and mixed media. Naturally occurring particulate matter exhibits a wide range of possible shapes and sizes. Even within specific genus of particle significant variability can occur. In addition, many naturally occurring aerosols have rough surfaces which are not regular and therefore exhibit significant scattering at random angles. Furthermore, although non-spherical particles may have various symmetry planes, they often tumble in flowing streams. This orientational variability coupled with size and shape variability assures that no two particles are positioned in precisely the same manner as they pass through the sample volume thereby preventing repeatability of the measurement. Small particles often form aggregates which can lack symmetry axes and can assemble in a myriad of different ways making particle generation a random process. These effects only serve to compound the variability of the TAOS measurement. Finally, complex aggregates may be comprised of multiple materials or, because of the packing fraction, demonstrate variable effective indices. Together all these factors lead to even greater variability in the observed TAOS images.

Despite these impediments, TAOS patterns from complex naturally occurring aerosols do exhibit characteristic features that may be analyzed in a statistical manner that provide insight into the nature of these ubiquitous and important particles. We have demonstrated that this is true by examining a series of pollen, spore, and powdered aerosols of significant variability, both in size and composition. The results presented herein indicate that novel parameters, which we felt evoked a sense of the physical characteristics of the particles, may be found within the seemingly random speckle pattern that can be used to classify. Other morphologically relevant features may exist and, perhaps, may be coaxed out of the data. Based on the relative success of the preliminary analysis, we believe that further exploration of new descriptors and more rigorous statistical analyses are worthwhile, and will be the subject of future work. An important factor for our approach, which allowed us to test these novel descriptors, is the implementation of multiple laser beams for spectral scattering and the ability to simultaneously acquire data without interference among the color channels. This too 
may be expanded to enhance the data and perhaps provide 620 useful information for inversion algorithms. The richness of the TAOS data provides a challenge, but not one that is insurmountable.

\section{Acknowledgements}

A portion of this work was performed under an SBIR Contract (W912HZ-06-C-0001) from the US Army Corps ${ }^{630}$ of Engineers Cold Regions Research and Engineering Lab while SH and SDF were with NovaWave Technologies (now ThermoFisher Scientific).

\section{References}

[1] M. Mishchenko, J. Hovenier, L. Travis (Eds.), Light Scattering by Nonspherical Particles: Theory, Measurements, and Appli- ${ }^{640}$ cations, Academic Press, San Diego, 1999.

[2] Y.-L. Pan, K. Aptowicz, R. Chang, M. Hart, J. Eversole, Characterizing and monitoring respiratory aerosols by light scattering, Optics Letters 28 (8) (2003) 589-591. doi:10.1364/0L.28. 000589

575 [3] K. Aptowicz, R. Pinnick, S. Hill, Y.-L. Pan, R. Chang, Optical scattering patterns from single urban aerosol particles at adelphi, maryland, usa: A classification relating to particle morphologies, Journal of Geophysical Research 111 (2006) D12212. doi:10.1029/2005JD006774

580 [4] P. Yang, Q. Feng, G. Hong, G. Kattawar, W. Wiscombe, M. Mishchenko, O. Dubovik, I. Laszlo, I. Sokolik, Modeling of the scattering and radiative properties of nonspherical dustlike aerosols, Journal of Aerosol Science 38 (2007) 995-1014. doi:10.1016/j.jaerosci.2007.07.001

[5] U. Krieger, C. Marcolli, J. Reid, Exploring the complexity of aerosol particle properties and processes using single particle techniques, Chemical Society Reviews 41 (2012) 6631-6662. doi: $10.1039 / \mathrm{c} 2 \operatorname{cs} 35082 \mathrm{c}$

[6] O. Sindoni, R. Saija, M. Iati, F. Borghese, P. Denti, G. Fer- ${ }^{660}$ nandes, Y.-L. Pan, R. Chang, Optical scattering by biological aerosols: experimental and computational results on spore simulants, Optics Express 14 (15) (2006) 6942-6950. doi: 10.1364/OE.14.006942

[7] J.-C. Auger, K. Aptowicz, R. Pinnick, Y.-L. Pan, R. Chang, ${ }^{665}$ Angularly resolved light scattering from aerosolized spores: observations and calculations, Optics Letters 32 (22) (2007) 33583360. doi:10.1364/0L.32.003358

[8] A. Wax, M. Giacomelli, T. Matthew, M. Rinehart, F. Robles, Y. Zhu, Optical spectroscopy of biological cells, Advances in ${ }^{670}$ Optics and Photonics 4 (3) (2012) 322-378. doi:10.1364/AOP. 4.000322

[9] S. Holler, Y.-L. Pan, R. Chang, J. Bottiger, S. Hill, D. Hillis, Two-dimensional angular optical scattering for the characterization of airborne microparticles, Optics Letters 23 (18) $(1998)^{675}$ 1489-1491. doi:10.1364/0L.23.001489

[10] S. Holler, J.-C. Auger, B. Stout, Y.-L. Pan, J. Bottiger, R. Chang, G. Videen, Observations and calculations of light scattering from clusters of spheres, Applied Optics 39 (36) (2000) 6873-6887. doi:10.1364/A0.39.006873

[11] S. Holler, M. Surbek, R. Chang, Y.-L. Pan, Two-dimensional angular optical scattering patterns as droplets evolve into clusters, Optics Letters 24 (17) (1999) 1185-1187. doi:10.1364/ OL.24.001185

[12] M. Barnes, K. Ng, K. Fukui, B. Sumpter, D. Noid, Prob- ${ }^{685}$ ing phase-separation behavior in polymer-blend microparticles: effects of particle size and polymer mobility, Macromolecules 32 (21) (1999) 7183-7189. doi:10.1021/ma990846r

[13] D. Secker, P. Kaye, R. Greenaway, E. Hirst, D. Bartley, G. Videen, Light scattering from deformed droplets and droplets ${ }^{690}$ with inclusions. i. experimental results, Applied Optics 39 (27) (2000) 5023-5030. doi:10.1364/A0.39.005023

14] G. Videen, W. Sun, Q. Fu, D. Secker, R. Greenaway, P. Kaye, Light scattering from deformed droplets and droplets with inclusions. ii. theoretical treatment, Applied Optics 39 (27) (2000) 5031-5039. doi:10.1364/A0.39.005031

[15] M. Barnes, S. Mahurin, B. Mehta, B. Sumpter, D. Noid, Three-dimensional photonic "molecules" from sequentially attached polymer-blend microparticles, Physical Review Letters 88 (2001) 015508. doi:10.1103/PhysRevLett.88.015508

[16] M. Summers, D. Burnham, D. McGloin, Trapping solid aerosols with optical tweezers: A comparison between gas and liquid phase optical traps, Optics Express 16 (11) (2008) 7739-7747. doi:10.1364/OE.16.007739

[17] K. Aptowicz, Y.-L. Pan, R. Chang, R. Pinnick, S. Hill, R. Tober, A. Goyal, T. Jeys, B. Bronk, Two-dimensional angular optical scattering patterns of microdroplets in the mid infrared with strong and weak absorption, Optics Letters 29 (17) (2004) 19651967. doi:10.1364/0L.29.001965

[18] M. Born, E. Wolf, Principles of Optics, 6th Edition, Pergamon Press, Oxford, 1980.

[19] G. Crosta, S. Zomer, Y.-L. Pan, S. Holler, Classification of single-particle two-dimensional angular optical scattering patterns and heuristic scatterer reconstruction, Optical Engineering 42 (9) (2003) 26892701. doi:10.1117/1.1599840

[20] S. Holler, S. Zomer, G. Crosta, Y.-L. Pan, R. Chang, J. Bottiger, Multivariate analysis and classification of two-dimensional angular optical scattering patterns from aggregates, Applied Optics 43 (33) (2004) 6198-6206. doi:10.1364/A0.43.006198

[21] G. Crosta, M. Camatini, S. Zomer, S. Holler, Y.-L. Pan, P. Bhaskara, P. Muangchareon, C. Sung, S. Cencetti, C. Regazzoni, Optical scattering (taos) by tire debris particles: preliminary results, Optics Express 8 (6) (2001) 302-307. doi: 10.1364/OE.8.000302

22] Z. Ulanowski, E. Hirst, P. Kaye, R. Greenaway, Retrieving the size of particles with rough and complex surfaces from two dimensional scattering patterns, Journal of Quantitative Spectroscopy \& Radiative Transfer 113 (18) (2012) 2457-2464. doi:10.1016/j.jqsrt.2012.06.019

[23] P. Kaye, J. Barton, E. Hirst, J. Clark, Simultaneous light scattering and intrinsic fluorescence measurements for the classification of airborne particles, Applied Optics 39 (21) (2000) 37383745. doi:10.1364/A0.39.003738

[24] K. Aptowicz, Y.-L. Pan, S. Martin, E. Fernandez, R. Chang, R. Pinnick, Decomposition of atmospheric aerosol phase function by particle size and asphericity from measurements of single particle optical scattering patterns, Journal of Quantitative Spectroscopy \& Radiative Transfer 131 (2013) 13-23. doi:10.1016/j.jqsrt.2013.03.020

[25] R. Johnson, D. Wichern, Applied Multivariate Statistical Analysis, 4th Edition, Prentice Hall, New Jersey, 1998.

[26] J.-C. Auger, G. Fernandes, K. Aptowicz, Y.-L. Pan, R. Chang, Influence of surface roughness on the elastic-light scattering patterns of micron-sized aerosol particles, Applied Physics B 99 (2010) 229-234. doi:10.1007/s00340-010-3914-0

[27] G. Crosta, Y.-L. Pan, K. Aptowicz, C. Casati, R. Pinnick, R. Chang, G. Videen, Automated classification of single airborne particles from two-dimensional angle-resolved optical scattering (taos) patterns by non-linear filtering, Journal of Quantitative Spectroscopy \& Radiative Transfer 131 (2013) 215-233. doi:10.1016/j.jqsrt.2013.06.009

[28] J. Mike, Classification Algorithms, Collins, London, 1985.

[29] W. Klecka, Discriminant Analysis, no. 19 in Quantitative Applications in the Social Sciences, Sage Publications, Thousand Oaks, CA, 1980.

[30] H. Shen, J. Carter, R. Brereton, C. Eckers, Discrimination between tablet production methods using pyrolysis-gas chromatography-mass spectrometry and pattern recognition, The Analyst 128 (2003) 287-292. doi:10.1039/B211574C

[31] S. Kachigan, Multivariate Statistical Analysis: A Conceptul Introduction, 2nd Edition, Radius Press, New York, 1991. 